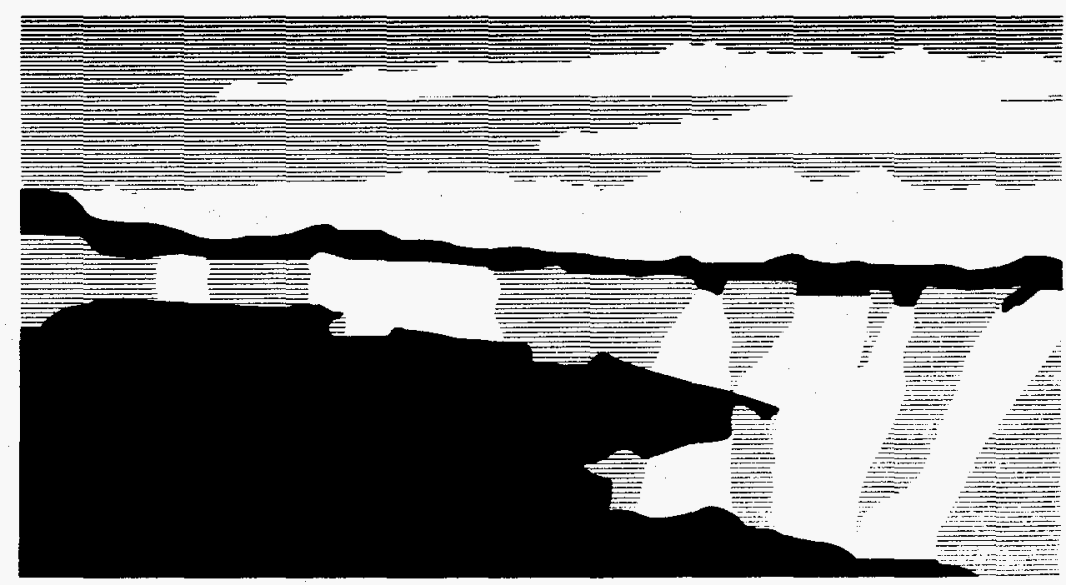

Los Alamos National Laboratory, an affirmative action/equal opportunity employer, is operated by the University of California for the U.S. Department of Energy under contract W-7405-ENG-36. By acceptance of this article, the publisher recognizes that the U.S. Government retains a nonexclusive, royalty-free license to publish or reproduce the published form of this contribution, or to allow others to do so, for U.S. Government purposes. The Los Alamos National Laboratory requests that the publisher identify this article as work performed under the auspices of the U.S. Department of Energy 


\section{DISCLAIMER}

This report was prepared as an account of work sponsored by an agency of the United States Government. Neither the United States Government nor any agency thereof, nor any of their employees, makes any warranty, express or implied, or assumes any legal liability or responsibility for the accuracy, completeness, or usefulness of any information, apparatus, product, or process disclosed, or represents that its use would not infringe privately owned rights. Reference herein to any specific commercial product, process, or service by trade name, trademark, manufacturer, or otherwise does not necessarily constitute or imply its endorsement, recommendation, or favoring by the United States Government or any agency thereof. The views and opinions of authors expressed herein do not necessarily state or reflect those of the United States Government or any agency thereof. 


\section{DISCLAMRER}

Portions of this doewenent may be Illegible in electronic image products. Images are produced from the best arailable original documenter 


\title{
RADIATION AND CRITICAL SHOCKS IN ATMOSPHERIC ENTRY
}

\author{
Gregory H. Canavan
}

Sub- and supercritical shock waves are produced during atmospheric entry. The radiation efficiency of the former increases strongly with velocity and altitude; that of the latter increases with altitude, but decreases with supercritical velocities. These efficiencies shift the region of maximum radiation one to two scale heights higher and decrease overall radiation efficiency.

A companion note discusses the radiation by hard objects during entry. ${ }^{1}$ That treatment left unanswered some issues, particularly in the radiation efficiency of large objects. This note addresses those issues in the context of the sub-and super-critical shock waves produced during their entry, which govern their overall radiation efficiency. In subcritical shocks, the radiation deposited in front of the shock does not raise the pre-shock temperature to the final value behind the shock. The temperature first rises due to the absorption of energy, then overshoots in the shock to a level, radiates strongly, and finally relaxes. The temperature of the radiating gas in the shock and that of the nonequilibrium radiating layer in front of it can be determined analytically, as can the radiative losses from them.

In supercritical shocks, radiation pre-heating of the in-flowing material is comparable to that by the shock itself. Thus, the radiation opacity of the inflowing material is significant, as is its radiation energy density relative to its thermal energy. However, the opaque region is selfregulating, and its radiation is limited by the critical temperature. At the critical velocity, the energy density in the preheat layer approaches that of the shock, which limits further increase. The critical velocity and temperature decrease with altitude due to the decrease of density, which progressively reduces the shock radiation efficiency until losses cause them to increase again.

The treatment of strong, planar shocks is applicable to the radiation from the shocks around entering objects, because fast, $\sim 1 \mathrm{~m}$ objects produce bow shocks thicker than photon mean free paths and smaller than its transverse extent. Corrections for shock thinning and loss at high altitudes are significant above $30-40 \mathrm{~km}$, where there is little radiation. Simplified expressions for temperatures and fluxes remain valid at the lower altitudes where most of the escaping radiation is generated.

The radiation efficiency of subcritical shocks increases strongly with velocity and altitude; that of supercritical shocks increases with altitude, but decreases with supercritical velocities, in contrast with conventional treatments, which assume a constant radiation efficiency or that the temperature saturates. Russian formulae have similar velocity dependencies similar to those for subcritical shocks. The effect of using critical shock efficiencies rather than constant 
efficiencies in entry calculations is to shift the region of maximum radiation one to two scale heights higher in altitude and to decrease overall radiation efficiency.

Shock structure and parameters. Figure 1 shows the Temperature T, density $\rho$, and pressure p profiles across a subcritical shock wave, ${ }^{2}$ i.e., one in which the radiation energy deposited in front of the shock does not raise the pre-shock temperature $T_{-}$to the final value $T_{1}$ behind the shock. In such a subcritical shock, the temperature first rises due to the absorption of the energy radiated by the shock, then overshoots in the shock to a level that radiates strongly, and finally relaxes to the jump value $T_{1}$. While the detailed structure of such shocks can be discussed analytically, the parameters of greatest interest here are the variation of $T$. and $T_{1}$ with shock speed $\mathrm{V}$, which determines the radiation loss and observables.

Figure 2 shows the variation of $T_{\text {. }}$ and $T_{1}$ with $V$. The variation of $T_{1}$ is somewhat stronger than linear; that of $T_{\text {. }}$ is much stronger. At V $=20 \mathrm{~km} / \mathrm{s}, T_{1}$ is about $4 \mathrm{ev}$-about an order of magnitude larger than $T$. Because $T$. increases more rapidly than $T_{1}$ with $V$, by $V \sim 90 \mathrm{~km} / \mathrm{s}$ the two temperatures are roughly equal at $\sim 25 \mathrm{ev}$.

Figure 3 shows the differences in the fluxes $F_{1}$ and $F$. At V $20 \mathrm{~km} / \mathrm{s}, F_{1}$ is about three orders of magnitude larger than $F_{\text {; }}$, however, $F_{-}$increases much more rapidly than $F_{1}$, so by $\sim 90$ $\mathrm{km} / \mathrm{s}$, the two are again about equal at $\sim 3 \times 10^{17} \mathrm{erg} / \mathrm{cm}^{2}-\mathrm{s}$.

Analysis of subcritical shocks. The variation of these temperatures and fluxes with velocity can be analyzed analytically. $T_{1}$ is within the optically thick region behind a strong shock, so the flux of radiation from it is $\mathrm{F}_{1} \sim \sigma \mathrm{T}_{1}^{4}$, where $\sigma \sim 10^{12} \mathrm{erg} / \mathrm{cm}^{2}-\mathrm{ev}^{4}-\mathrm{s}$ is the StefanBoltzmann constant. The temperature can be determined approximately from the approximate formula $\mathrm{V}^{2} / 2 \sim \mathrm{bT}_{1}^{3 / 2}$, where $\mathrm{b} \sim 33 \mathrm{~km}^{2} / \mathrm{s}^{2}-\mathrm{ev}^{3 / 2}$, which produces ${ }^{3}$

$$
\mathrm{T}_{1} \sim\left(\mathrm{V}^{2} / 2 \mathrm{~b}\right)^{2 / 3}
$$

from which $T_{1} \sim V^{4 / 3}$, in accord with the scaling in Fig. 2. Substituting this result into $F_{1}$ produces

$$
\mathrm{F}_{1} \sim \sigma \mathrm{T}_{1}^{4} \sim \sigma\left(\mathrm{V}^{2} / 2 \mathrm{~b}\right)^{8 / 3} \sim \mathrm{V}^{16 / 3} \text {, }
$$

in accord with the scaling of $F_{1}$ seen in Fig. 3. The principal result of interest here is that the radiation flux $F_{1}$ is large, that it scales as $\sim V^{16 / 3}$, and that most of it is reabsorbed within a few mean free paths, preheating the incoming air to $T$. Only a small fraction of $F_{1}$ escapes to long ranges, as discussed further below.

Preheat layer. The energy flux at $T_{\text {. }}$ is dominated by kinetic rather than radiation energy. The flux can be estimated with Eq. (2) and an approximate equation of state. Figure 4 shows the specific energy density in front of the shock, e, as a function of temperature, together with $\mathrm{e} / \mathrm{T}_{-}^{3 / 2}$ $\sim$ constant $\mathrm{K}$, which is $\sim 10 / \mathrm{molecule}^{\mathrm{e} \mathrm{v}^{1 / 2}}$ or $\sim 3 \times 10^{11} \mathrm{erg} / \mathrm{g}-\mathrm{ev}^{1 / 2}$ in the region from 0.8 to $20 \mathrm{ev}$ of primary interest. With this approximate equation of state, the equality of the outward flux of radiation and the inward flux of thermal energy resulting from its absorption in the cold gas gives a conservation relationship at the shock of 


$$
\rho \mathrm{VKT}^{3 / 2} \sim \mathrm{F}_{1} \sim \sigma \mathrm{T}_{1}^{4} \sim \sigma\left(\mathrm{V}^{2} / 2 \mathrm{~b}\right)^{8 / 3}
$$

which can be solved for

$$
\text { T. } \sim\left[\sigma\left(V^{2} / 2 b\right)^{8 / 3} / \rho V K\right]^{2 / 3} \sim V^{26 / 9} / \rho^{2 / 3},
$$

This result shows that $T$. increases almost as strongly as $\mathrm{V}^{3}$ - much more rapidly than $T_{1}$, as seen in Fig. 2. The approximate Eqs. (1) and (3) were used to generate Figs. 1 and 2, which agree with results in the literature from more detailed treatments.

Critical velocity and temperature. For $V \approx 90 \mathrm{~km} / \mathrm{s}$ at sea level, Fig. 2 shows that, the pre- and post- shock temperatures converge to about $25 \mathrm{ev}$, and Fig. 3 shows that the pre- and post- shock fluxes converge to about $3 \times 10^{17} \mathrm{erg} / \mathrm{cm}^{2}-\mathrm{s}$, which provides several ways of estimating the critical velocity $V_{c}$. Perhaps the simplest is to equate $F_{1} \sim \sigma T_{1}^{4} \sim \sigma\left(V^{2} / 2 b\right)^{8 / 3}$ to the flux of kinetic energy, $1 / 2 \rho \mathrm{V}^{3}$, and solve for

$$
V_{c}=\left[(2 b)^{8 / 3} \rho / 2 \sigma\right]^{3 / 7},
$$

which depends only on density. $V_{c}$ can be substituted into $F_{1}$ to determine the critical temperature Both are shown as functions of altitude in Fig. 5. The critical temperature falls from $\sim 25 \mathrm{ev}$ at sea level to $1 \mathrm{ev}$ at $40 \mathrm{~km}$. The critical velocity falls from about $90 \mathrm{~km} / \mathrm{s}$ at sea level to $8 \mathrm{~km} / \mathrm{s}$ at $40 \mathrm{~km}$. While in standard density air, the transition from subcritical to supercritical shocks takes place at velocities too high to be of concern, at the altitudes at which $\sim 1 \mathrm{~m}$ objects enter the atmosphere, the transition occurs at typical entry velocities.

The physical basis for the transition is clear from the fact that the temperature behind the shock, $T_{1} \sim\left(V^{2} / 2 b\right)^{2 / 3}$, and hence the flux $F_{1}$, are independent of density, as long as the shock is thick. However, the flux of kinetic energy is proportional to $\rho$. Thus, as the density falls, the ratio of radiation to kinetic flux increases, which produces criticality with lower velocities and temperatures at higher altitudes.

Radiation losses from the preheat layer. The flux of kinetic energy into the shock is on the order of $\rho V^{3} / 2$. A fraction of this flux is retained in the heated gas behind the shock; a fraction $f_{1}$ is radiated from the shock; and a fraction $f_{-}$is radiated from the preheated gas in front of the shock. The last two can be estimated analytically; the first is then determined as a residual. The fraction radiated from the preheated region can be determined simply. That region has an optical thickness of a few mean free paths, so it radiates as a blackbody with the temperature $T_{\text {. } ~}$ $\left[\sigma\left(\mathrm{V}^{2} / 2 \mathrm{~b}\right)^{8 / 3} / \mathrm{VK}\right]^{2 / 3}$ of Eq. (4), which produces a normalized flux

$$
\text { f. } \sim \sigma\left[\sigma\left(V^{2} / 2 b\right)^{8 / 3} / V K\right]^{8 / 3} / \rho V^{3} / 2 \sim V^{8.6} / \rho^{11 / 3},
$$

to long distances. For $V$ small, $f_{-}$is small, but for $V \sim 90 \mathrm{~km} / \mathrm{s}$, it approaches the total flux of kinetic energy, as shown by Fig. 6 , which gives the normalized fluxes $\mathrm{f}_{1}$ and $\mathrm{f}$. as functions of $\mathrm{V}$. At $10 \mathrm{~km} / \mathrm{s}, F_{1}$ is about $1 \%$ of the flux of kinetic energy $\rho V^{3} / 2$, but $f_{-}$is only $\sim 10^{-8}$. By V $=30$ $\mathrm{km} / \mathrm{s}, \mathrm{f}_{1}$ is about $10 \%$ and $\mathrm{f}_{\text {. }}$ is about $10^{-4}$. By $\mathrm{V}=50 \mathrm{~km} / \mathrm{s}, \mathrm{f}_{1}$ is about $30 \%$, and $\mathrm{f}$. is about $1 \%$. By 
$90 \mathrm{~km} / \mathrm{s}$, the radiation fluxes approximately equal the kinetic fluxes. Radiation at higher velocities is discussed in the subsequent section on super-critical shocks.

Radiation from the shock layer. As shown in Fig. 6, the radiation from the shock layer is a significant fraction of the incident kinetic energy flux even when the shock velocity is only $1-10 \%$ of the critical velocity. However, the strong re-absorption by the preheated layer in a subcritical shock strongly reduces the fraction of radiation that escapes to long range. That fraction can be estimated by using the fact that cold air is relatively transparent to photons with energies below 4-5 ev and opaque to photons with larger energies. The air behind the shock is thick and in equilibrium, so it radiates a black body spectrum $B(w, T)$ with temperature $T_{1}$. The fraction that escapes is

$$
\varepsilon_{1}=\int_{0}^{w_{0}} \mathrm{dw} \mathrm{B}\left(\mathrm{w}, \mathrm{T}_{1}\right) / 4 \sigma \mathrm{T}_{1} / \mathrm{c},
$$

where $w_{0}$ is the cutoff photon energy, which is varied from 3 to $5 \mathrm{ev}$ in Fig. 7. The fraction of energy escaping increases about linearly with cutoff energy and decreases strongly with increasing shock temperature. For $\mathrm{w}_{\mathrm{o}}$ very small, $\mathrm{f}_{1}$ would vary rigorously as $1 / \mathrm{T}^{3}$. The actual variation over the 1-10 ev range of interest can be approximated by the $1 / \mathrm{T}^{2}$ curve sketched on the figure. Saturation at small temperatures, where all radiated energy escapes, can be treated by an interpolation formula of the form

$$
\varepsilon_{1}=1 /\left(1+\mathrm{a} \mathrm{T} \mathrm{T}^{2}\right)
$$

where $\mathrm{a} \sim 1 / \mathrm{ev}^{2}$. Thus, the net radiation from the shock can be approximated by

$$
\Delta \mathrm{F}_{1} \approx \sigma \mathrm{T}_{1}^{4} /\left(1+\mathrm{a} \mathrm{T}^{2}\right) \sim \sigma \mathrm{T}_{1}^{2} / \mathrm{a},
$$

where the latter form holds for the high velocities of greatest interest. Figure 8 shows the

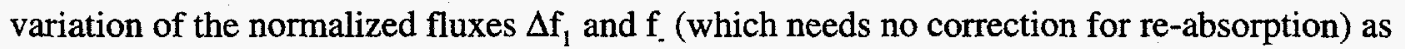
functions of $V$. The net flux $\Delta f_{1}+f_{\text {f }}$ is constant below $V \sim 40 \mathrm{~km} / \mathrm{s}$ and rises rapidly with $\mathrm{f}_{-}$for larger V. At small V, where $\Delta F_{1}$ is dominant, substituting Eq. (1) into Eq. (9) shows that the normalized net flux varies as

$$
\Delta \mathrm{f}_{1} \sim \sigma \mathrm{T}_{1}^{2} / \mathrm{a} \rho \mathrm{V}^{3} \sim \sigma\left[\left(\mathrm{V}^{2} / 2 \mathrm{~b}\right)^{2 / 3}\right]^{2} / \mathrm{a} \rho \mathrm{V}^{3} \sim 1 / \rho \mathrm{V}^{1 / 3},
$$

which gives the slow variation of $\Delta f_{1}$ seen in Fig. 8. For large $V$, the scaling of the total flux is essentially that of $f_{\text {., }}$ as discussed above. The result of strong re-absorption of radiation from the shock is to make it dominant only for low velocities, where it is $\sim 0.3 \%$ of the overall flux. Note, however, that $\Delta \mathrm{f}_{1} \sim 1 / \mathrm{p}$, so it is necessary to examine the variation of this balance with altitude.

Variation of radiation with altitude. The critical velocity as a function of altitude is given by $\mathrm{Eq}$. (5), from which the critical temperature is

$$
T_{c} \sim\left(V_{c}{ }_{c}^{2} / 2 b\right)^{2 / 3} \sim\left\{\left[(2 b)^{8 / 3} \rho / 2 \sigma\right]^{6 / 7} / 2 b\right\}^{2 / 3} \sim\left[2 b^{3}(\rho / \sigma)^{2}\right]^{2 / 7} \sim \rho^{4 \pi},
$$

Figure 9 shows the decrease of $T_{c}$ with altitude, together with the preheat layer temperatures for shocks at $V=10,20$, and $30 \mathrm{~km} / \mathrm{s}$. For $V=10 \mathrm{~km} / \mathrm{s}$, the intersection of $T$. and $T_{c}$ occurs at about $35 \mathrm{~km}$, where $T_{-}=T_{c} \sim 1.3 \mathrm{ev}$, which is also the value of $T_{1}$ for $10 \mathrm{~km} / \mathrm{s}$, which does not vary 
with altitude. For $V=20 \mathrm{~km} / \mathrm{s}$, the intersection occurs at about $25 \mathrm{~km}$. For $\mathrm{V}=30 \mathrm{~km} / \mathrm{s}$, it occurs at about $18 \mathrm{~km}$. Crudely, increasing the velocity $10 \mathrm{~km} / \mathrm{s}$ drops the critical altitude by about a scale height.

The physical significance of these intersections is that for $\mathrm{V}=10 \mathrm{~km}$, the subcritical scaling would predict that the preheated layer could radiate more energy than is in the shock at altitudes above about $35 \mathrm{~km}$, which is physically impossible. To find the appropriate limit for high velocities, it is necessary to briefly review supercritical shocks.

Supercritical shocks are those in which the radiation heating of the material flowing into the shock is comparable to the heating in the shock itself, i.e., $T_{-} \sim T_{1}$. In this situation the radiation opacity of this inflowing material is significant, as is its radiation energy density relative to its thermal energy. It is possible to study the structure of such shocks analytically, but the main result needed for this discussion is the result that the opaque region is essentially selfregulating. ${ }^{4}$ That is, it adjusts its optical depth such that the radiation flow from the shocked region at $T_{1}$ to the critical surface at $T_{c}$ where the optical thickness drops to unity is just adequate to allow the critical surface to radiate the incident kinetic energy.

In this case the critical surface is defined as the surface in front of the discontinuity at which the thermal and radiation energy densities and fluxes are comparable. The former is given by Eq. (3) as $\rho \mathrm{VKT}_{\mathrm{c}}{ }^{3 / 2}$, where $\mathrm{V} \sim \mathrm{kT}_{\mathrm{c}}{ }^{1 / 2}$, and the latter is $\sim \sigma \mathrm{T}_{\mathrm{c}}{ }^{4}$. Equating these results gives

$$
\operatorname{\rho kKT}_{\mathrm{c}}{ }^{2}=\sigma \mathrm{T}_{\mathrm{c}}^{4} \text {, }
$$

which can be solved for

$$
T_{c}=(\rho k K / \sigma)^{1 / 2},
$$

although the specific exponent depends on the fit used for the equation of state. The principal dependence is on $\rho^{1 / 2}$, which is essentially the same as the $\rho^{4 / 7}$ of Eq. (11). Figure 10 contrasts the variation of $T_{c}$ with $\rho$ from the supercritical solution with that from the subcritical solution of Eq. (11). There is little difference at low altitude; at most a factor of two a high altitude. Most of this is due to the differing fits to the equation of state used. Both curves indicate a simple log-linear decrease with altitude, with the $T_{c}$ from the supercritical solution lying slightly higher because of its smaller (square root) exponent. In the supercritical case the radiation loss is

$$
\sigma \mathrm{T}_{\mathrm{c}}^{4} \sim \rho \mathrm{kKT}_{\mathrm{c}}^{2} \sim \rho \mathrm{kKK}(\rho \mathrm{kK} / \sigma) \sim(\mathrm{\rho kK})^{2} / \sigma,
$$

which at sea level is $\sim\left(10^{-3} \mathrm{~g} / \mathrm{cc} \times 3 \times 10^{6} \mathrm{~cm} / \mathrm{s}-\sqrt[V]{\mathrm{ev}} \times 3 \times 10^{11} \mathrm{erg} / \mathrm{g}-\mathrm{ev}^{3 / 2}\right)^{2} / 10^{12} \mathrm{erg} / \mathrm{cm}^{2}-\mathrm{ev}^{4}-\mathrm{s} \sim$ $6 \times 10^{17} \mathrm{erg} / \mathrm{cm}^{2}$-s, corresponding to a temperature of $\sim 23 \mathrm{ev}$, although the flux falls as $\rho^{2}$ with altitude. Supercritical shocks can radiate a great deal of energy at sea level, but lose the capacity to do so rapidly with altitude. Their energy loss relative to the influx of kinetic energy varies as

$$
f_{s} \sim(\rho k K)^{2} / \sigma / \rho V^{3} / 2 \sim \rho / V^{3},
$$

which falls rapidly with altitude and velocity. Since the $T_{c}$ of Eq. (13) is similar to that of Eq. (11), Fig. 9 again applies. $T_{c}$ is shifted to slightly higher values, which shifts the crossings to 
slightly higher altitudes. However, that is a minor consideration. The meaning of the crossing of the $T_{\text {. }}$ and $T_{c}$ curves is now clear. As long as $T_{.}<T_{c}$, the radiative losses are $\sigma T_{-}^{4}$, as assumed in constructing Fig. 9. When $T_{.}=T_{c}$, the optical depth of the preheat layer exceeds unity, and the $T_{-}$

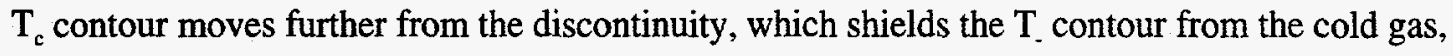
reducing radiation transport and losses. For higher altitudes, the radiating temperature no longer continues to follow $T$, which approaches $T_{1}$, instead it falls along $T_{c}$, reflecting the maximum flux that the critical surface can radiate.

Figure 11 shows the resulting temperature, $T$. limited by $T_{c}$, as a function of altitude for $V$ $=10,20$, and $30 \mathrm{~km} / \mathrm{s}$. The temperature for $10 \mathrm{~km} / \mathrm{s}$ peaks at about $1.3 \mathrm{ev}$ at $35 \mathrm{~km}$ and then falls. Those for 20 and $30 \mathrm{~km} / \mathrm{s}$ peak at about 3 and $5 \mathrm{ev}$ at 25 and $20 \mathrm{~km}$, respectively. The limited radiation from the supercritical shock effectively eliminates the high temperatures predicted in Fig. 9 at high altitudes. Figure 12 shows the resulting normalized fluxes corresponding to these temperatures. The flux for $10 \mathrm{~km} / \mathrm{s}$ peaks at about 0.55 at $35 \mathrm{~km}$, the altitude of the peak temperature, and then falls rapidly to the $0.3 \%$ leakage from the shock by about $20 \mathrm{~km}$. The flux for $20 \mathrm{~km} / \mathrm{s}$ peaks at about 0.95 at $25 \mathrm{~km}$ and then falls to the shock leakage by about $10 \mathrm{~km}$. The flux for $30 \mathrm{~km} / \mathrm{s}$ peaks at about 0.7 at $20 \mathrm{~km}$ and falls to the shock leakage by about $5 \mathrm{~km}$. The overall effect of the increase of $T_{-}$and $F_{-}$with altitude and the decrease of $T_{c}$ and $F_{c}$ with altitude is to produce a sharp peak in the radiated flux at the altitude at which $T_{-}$and $=T_{c}$. Thus, calculating the radiative loss at a given altitude is a matter of calculating $T_{1}, T_{-}, T_{c}$; testing whether $T_{.}<T_{c}$; and using $T_{-}$in calculating the radiative loss if it is and using $T_{c}$ if not.

Connection to entering objects. The discussion above has treated strong, planar shocks at various altitudes. These results are directly applicable to the radiation from the shocks that form around entering objects. An object of diameter $\mathrm{D}$ produces a bow shock whose thickness and width are both of order $\mathrm{D}$. For $\mathrm{a} \sim 1 \mathrm{~m}$ object, the thickness of the shock might be on the order of $10-20 \mathrm{~cm}$. In the shock from an object moving at $15 \mathrm{~km} / \mathrm{s}$ at $20 \mathrm{~km}$, the photon mean free path, $\mathrm{L}$, is a few centimeters, so $\mathrm{L} \ll \mathrm{D}$. Thus, the fact that the object is curved does not lessen the validity of the assumption that the flow is planar.

The relaxation processes sketched in Fig. 1 occur over a few mean free paths, so they occur over a distance $<D$. Thus, the fact that the flow must turn at the surface of the object does not disturb the calculation of the outward flow of radiation, so long as the shock is optically thick. For shocks that are not optically thick, their radiation density and flux are depressed in proportion to $\mathrm{D} / \mathrm{L}$, which can be accommodated by dividing the black body flux by $1+\mathrm{L} / \mathrm{D}$, which produces an effective radiation flux of

$$
\mathrm{F}_{\text {eff }} \sim \sigma \mathrm{T}^{4} /(1+\mathrm{L} / \mathrm{D})
$$

that reduces to $\sigma \mathrm{T}^{4}$ for black bodies and $(\mathrm{D} / \mathrm{L}) \sigma \mathrm{T}^{4}$ for thin ones. 
Geometry. A second correction for optical thickness involves the geometry of redeposition of thermal radiation. It is assumed in deriving Eq. (12) that essentially all of the energy radiated from the shock is reabsorbed by the cold or heated gas in front of it. That approximation is obviously valid for a planar shock, apart from the small fraction that escapes to infinity, as there is nowhere else for the radiation to go. If emitted radiation is deposited isotropically a distance $L$ from an object of diameter $D$, the probability that it will lie in the path of the object and hence be recovered is $\sim(D / L)^{2}$; the rest of the radiation is effectively lost. This geometric dilution can be accounted for by dividing the emitted flux by $(1+\mathrm{L} / \mathrm{D})^{2}$ to produce an adjusted effective flux

$$
F_{\text {eff' }} \sim \sigma T^{4} /(1+L / D)^{3}
$$

which includes the corrections for both shock thickness and geometric loss. With these two corrections, Eq. (12) becomes

$$
\sigma \mathrm{T}_{\mathrm{c}}^{4} \sim(1+\mathrm{L} / \mathrm{D})^{3} \rho k K \mathrm{~T}_{\mathrm{c}}{ }^{2}
$$

Since $\mathrm{L}$ is an exponential function of $\mathrm{T}$, this equation is transcendental, but it can be solved simply by iteration. Fig. 13 shows the resulting $T_{c}$ as a function of altitude for various object diameters. The curves follow the supercritical $T_{c}$ curve of Fig. 10 to about $20 \mathrm{~km}$ altitude for all D. The curve for $\mathrm{D}=0.25 \mathrm{~m}$ has a minimum of $\sim 7 \mathrm{ev}$ at $25 \mathrm{~km}$ and then climbs sharply to $\sim 20$ $\mathrm{ev}$ by $40 \mathrm{~km}$. The curve for $1 \mathrm{~m}$ has a minimum of $\sim 5 \mathrm{ev}$ at $30 \mathrm{~km}$ and then climbs to $\sim \mathrm{ev}$ by $40 \mathrm{~km}$. That for $4 \mathrm{~m}$ has a minimum of $\sim 3.5 \mathrm{ev}$ at $35 \mathrm{~km}$. Thus, the corrections of $T_{c}$ for shock thickness and geometric loss are small for altitudes below $20 \mathrm{~km}$ and large for altitudes above 30 $\mathrm{km}$, where the corrected $T_{\mathrm{c}} \mathrm{s}$ increase rather than falling with altitude.

As an example of the use of Fig. 13, a $1 \mathrm{~m}$ object entering vertically at about $30 \mathrm{~km} / \mathrm{s}$ would have a shock temperature of about $6 \mathrm{ev}$; thus, its radiation would not be limited above about $38 \mathrm{~km}$. At that altitude it would reach criticality, and its radiating temperature would be reduced, to about $5 \mathrm{ev}$ at $30 \mathrm{~km}$. Then at about $23 \mathrm{~km}$ its temperature would again drop below critical. It would not be limited at lower altitudes. The reduction would be larger for larger objects, e.g., a $4 \mathrm{~m}$ object at $30 \mathrm{~km} / \mathrm{s}$ would have its radiating temperature reduced by a factor of $\sim(3.5 / 6)^{4} \sim 0.12$ at $35 \mathrm{~km}$.

The critical velocity $V_{c} \sim k \sqrt{ } T_{c}$ is shown as a function of altitude for these diameter objects in Fig. 14. The shape is similar to that of Fig. 13, although the extremes are suppressed. The overall variation of $V_{c}$ for large objects is from $\sim 180 \mathrm{~km} / \mathrm{s}$ at low altitudes to $\sim 60 \mathrm{~km} / \mathrm{s}$ at $35 \mathrm{~km}$, or about a factor of three. The variation for smaller objects is about a factor of 2 . For a large object entering at $\mathrm{V} \sim 30 \mathrm{~km} / \mathrm{s}$, the ratio of $\mathrm{V} / \mathrm{V}_{\mathrm{c}}$ would be $\sim 2$. From Eq. (6),

$$
\mathrm{f} \sim 1.7\left(\mathrm{~V} / \mathrm{V}_{\mathrm{c}}\right)^{8.6} \rho_{\mathrm{o}} / \rho
$$


from which for a large object entering at $\mathrm{V} \sim 30 \mathrm{~km} / \mathrm{s}$, the radiating efficiency would be $2(1 / 2)^{8.6} \sim 0.5 \%$ at sea level and about $50 \%$ at $15 \mathrm{~km}$, where the bulk of the radiation would be generated. The former is only slightly above the $\sim 0.3 \%$ leakage from the shock.

The reason for the minimum in $V_{c}$ and $T_{c}$ and is straightforward. Figure 15 shows $D / L$ as a function of altitude for the values of $D$ above. It shows that the shocks for the $0.25,1$, and $4 \mathrm{~m}$ objects are thick for altitudes above 30,35 , and $40 \mathrm{~km}$, respectively, and thin for higher altitudes. When $D / L$ is large, the correction in Eq. (18) is small and $T_{c} \sim \sqrt{ }\left(\rho k K_{c}{ }_{c}^{2} / \sigma\right)$, as in the planar case studied earlier in Eq. (13). When $D / L$ is small, $T_{c}{ }^{2} \sim(L / D)^{3} V(\rho k K / \sigma)$, so $T_{c}$ increases by a large factor which in turn increases exponentially with density.

Because the corrections are significant only at altitudes above $30-40 \mathrm{~km}$, where there is little radiation, the temperatures and fluxes of Figs. 11 and 12 remain valid at the lower altitudes where most of the escaping radiation is generated.

Heat flux to object. In calculating the radiation loss, the results of planar shock theory can be used directly. However, if it is necessary to calculate the radiative flux to the body, the deflection of the flow and hence the finite diameter of the object must be taken into account. For that calculation it is not the competition of preheat and supercriticality that must be considered, rather it is the simple radiative diffusion of heat from the inner shock at temperature $T$. to the solid object of effective temperature $\sim 0.3-0.4$ ev corresponding to the evaporation temperature of the object material at the elevated temperature and pressure of the shock.

Comparison with other treatments. The main scaling results of the results above are the estimates of the radiation efficiency of subcritical shocks of $\sim V^{26 / 9} / \rho^{2 / 3}$ in Eq. (4) and of the radiation efficiency of supercritical shocks of $\sim \rho^{47} / \rho \mathrm{V}^{3} \sim 1 / \rho^{3 /} \mathrm{V}^{3}$ in Eq. (11). The former gives a strong increase with object velocity and altitude; the latter gives a strong increase with altitude, but decrease with velocity $\mathrm{V}>\mathrm{V}_{\mathrm{c}}$. Conventional treatments assume a constant reradiation efficiency for simplicity, although there is no strong justification for doing so. ${ }^{5}$ Other treatments assume that the temperature saturates at a value of $2-3 \mathrm{ev}$, which is not consistent with the strong increase of temperature with altitude for subcritical shocks and decrease for supercritical shocks.

${ }^{6}$ Russian interpolation formulae have velocity dependencies not unlike those for subcritical shocks and make reference to similar distinctions between subcritical and supercritical shocks. ${ }^{7}$ The overall effect of using critical shock efficiencies rather than constant efficiencies in performing entry calculations is to shift the region of maximum radiation one to two scale heights higher in altitude and to decrease the overall radiation efficiency.

Summary and conclusions. An earlier note discussed the radiation by hard objects during entry, leaving unanswered some issues in the radiation efficiency of large objects, which are addressed in the context of the sub-and super-critical shock waves they produce during entry. In a subcritical shock wave the radiation deposited in front of the shock does not raise the pre- 
shock temperature to the final value behind the shock. The temperature first rises due to the absorption of energy, then overshoots in the shock to a level, radiates strongly, and finally relaxes to the jump value. The temperature of the radiating gas in the shock and that of the nonequilibrium radiating layer in front of it can be determined analytically, as can the radiative losses from them.

In supercritical shocks, radiation pre-heating of the in-flowing material is comparable to that by the shock itself. Thus, the radiation opacity of the inflowing material is significant, as is its radiation energy density relative to its thermal energy. However, the opaque region is essentially self-regulating, and its radiation is limited by the critical temperature. At the critical velocity, the energy density in the preheat layer approaches that of the shock, which limits further increase. The critical velocity and temperature decrease with altitude due to the decrease of density, which progressively reduces the shock radiation efficiency until altitudes of 30-40 $\mathrm{km}$, above which losses cause the critical velocity and temperature to increase again.

The discussion of strong, planar shocks is applicable to the radiation from the shocks around entering objects, because $\sim 1 \mathrm{~m}$ objects produce bow shocks a few tens of $\mathrm{cm}$ in thickness and a few meters in width. Photon mean free paths are a few centimeters, so the fact that the shock is a few tens of $\mathrm{cm}$ thick does not lessen the validity of the assumption that it is infinitely thick and the fact that the object is curved on the scale of a few meters does not lessen the validity of the assumption that the flow is planar on the scale of a mean free path. It is, however, necessary to correct for the thinning of the shock and geometric loss of energy at high altitudes. Because the corrections are significant only at altitudes above $30-40 \mathrm{~km}$, where there is little radiation, the simplified temperatures and fluxes remain valid at the lower altitudes where most of the escaping radiation is generated.

The main scaling results are the estimates of the radiation efficiencies of subcritical and supercritical shocks as functions of object altitudes and velocity. The former increases strongly with velocity and altitude; the latter increases with altitude, but decreases with supercritical velocities. Conventional treatments assume a constant radiation efficiency for simplicity, or assume that the temperature saturates at a few ev, which is not consistent with the strong increase of temperature with altitude for subcritical shocks and decrease for supercritical shocks. Russian formulae have similar velocity dependencies similar to those for subcritical shocks. The effect of using critical shock efficiencies rather than constant efficiencies in entry calculations is to shift the region of maximum radiation one to two scale heights higher in altitude and to decrease overall radiation efficiency. 


\section{References}

1. G. Canavan, "Detailed Treatment of Radiation from Entering Hard Objects," Los Alamos report LA-UR-97-928, March 1997.

${ }^{2}$. Y. Zel'dovich and Y. Raizer, Physics of Shock Waves and High-Temperature Hydrodynamic Phenomena (Academic Press, New York and London, 1967).

3. Y. Zel'dovich and Y. Raizer, Physics of Shock Waves and High-Temperature Hydrodynamic Phenomena, op. cit., p. 536, Table 7.4.

${ }^{4}$. Y. Zel'dovich and Y. Raizer, Physics of Shock Waves and High-Temperature Hydrodynamic Phenomena p. cit., pp. 539-543.

5. J. Hills and M. Goda, "The Fragmentation of Small Asteroids in the Atmosphere," Astronomical Journal, Vol. 105, No. 3, March 1993, pp. 1114-1144.

6. C. Chyba, P. Thomas, and K. Zahnle, "The 1908 Tunguska explosion: atmospheric disruption of a stony asteroid," Nature, Vol. 361, 7 January 1993, pp. 40-44.

?. I. Nemtchinov, "Large Meteoroids Impact onto the Earth's Atmosphere," Sandia National Laboratory Final Report Part II, 17 April 1977. 

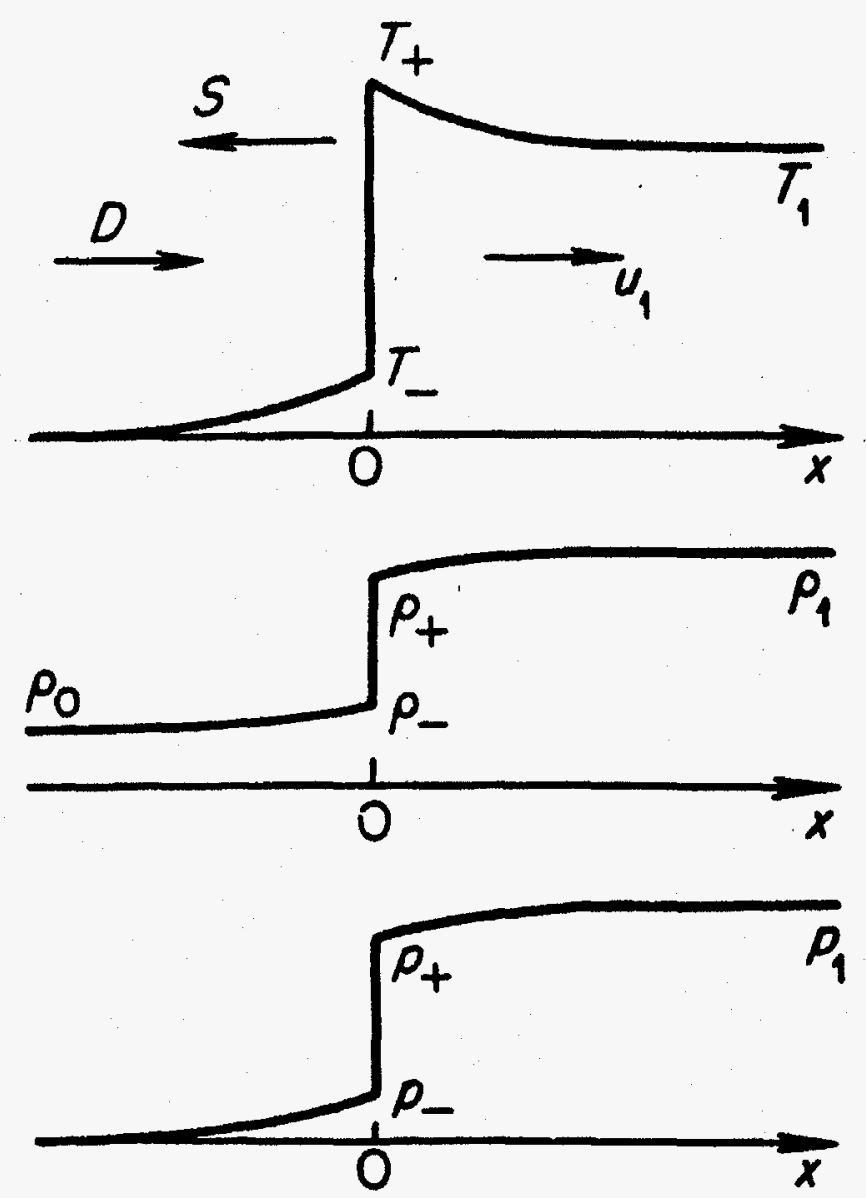

Fig. 1. Temperature, density, and pressure profiles in a shock front of not too large a strength, taking into account radiant heat exchange. 
(s/ury) pords yoous

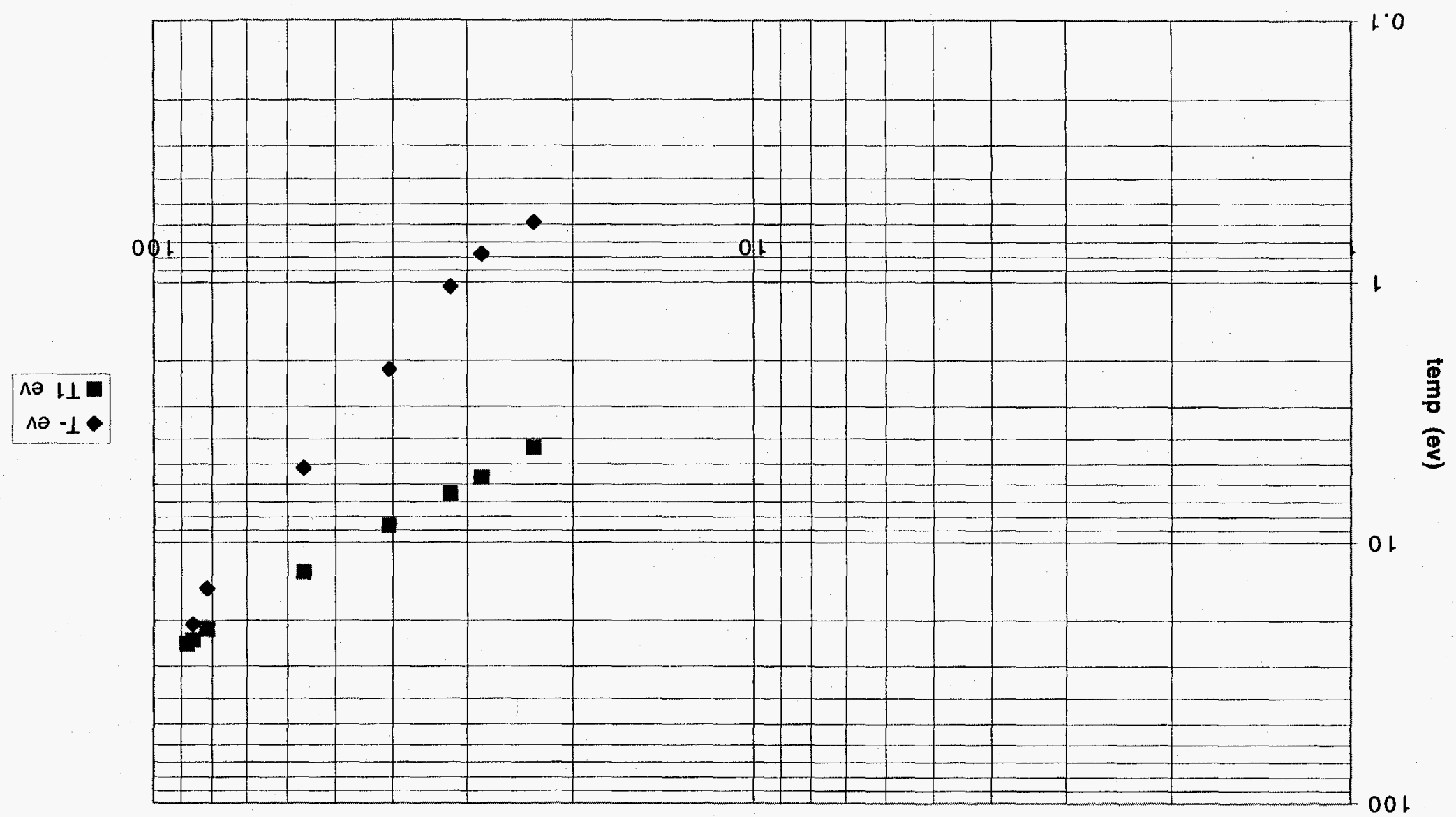

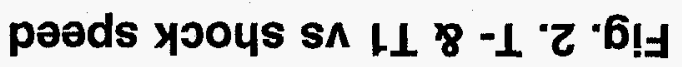

O s^ sdmə」 乙む 
F3 flux $v V$

\section{Fig. 3. Fluxes vs shock speed}

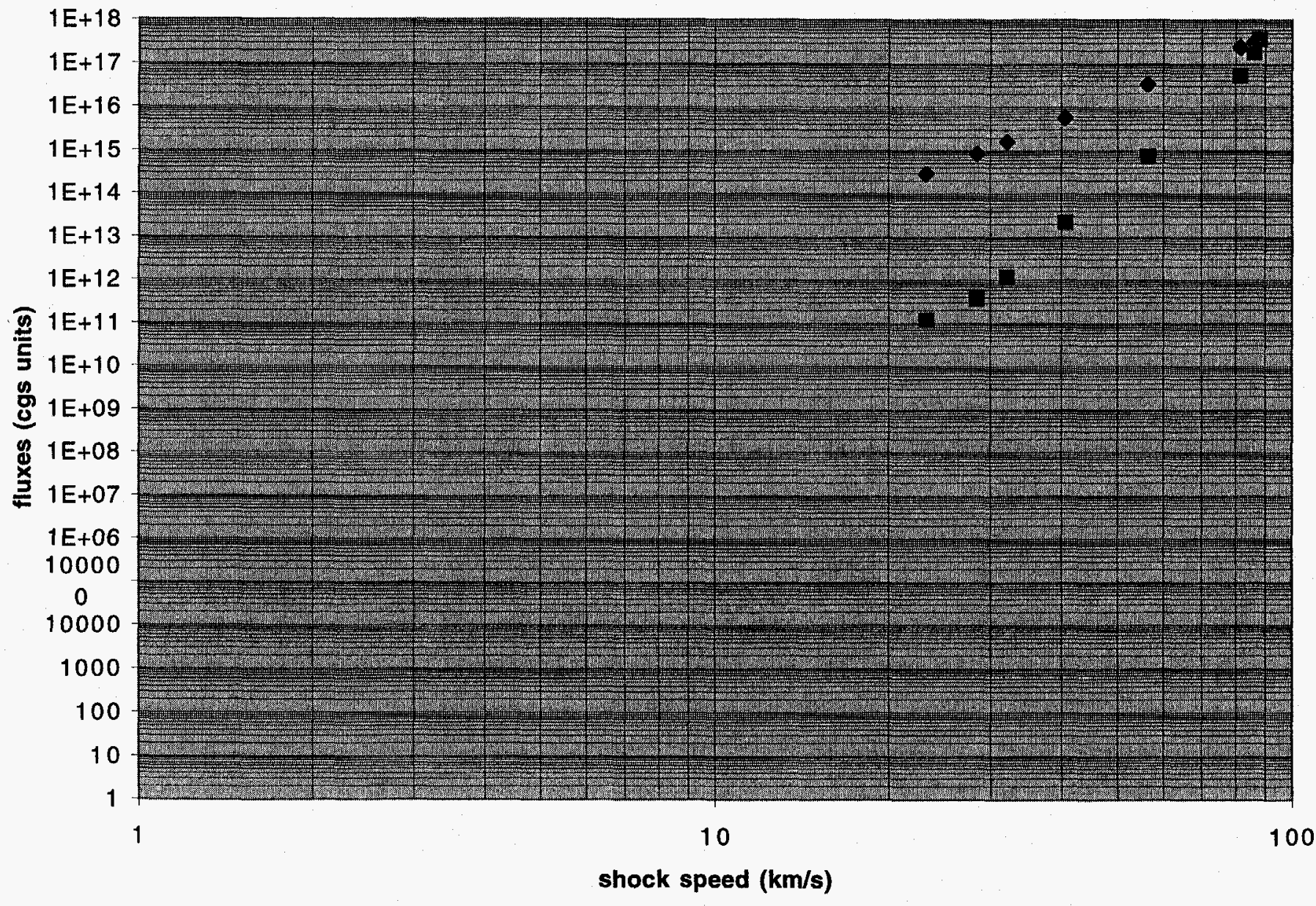

- flux e/cm2s

min flux- 
F4 epg $\vee T$ -

Fig. 4. Energy density vs T-

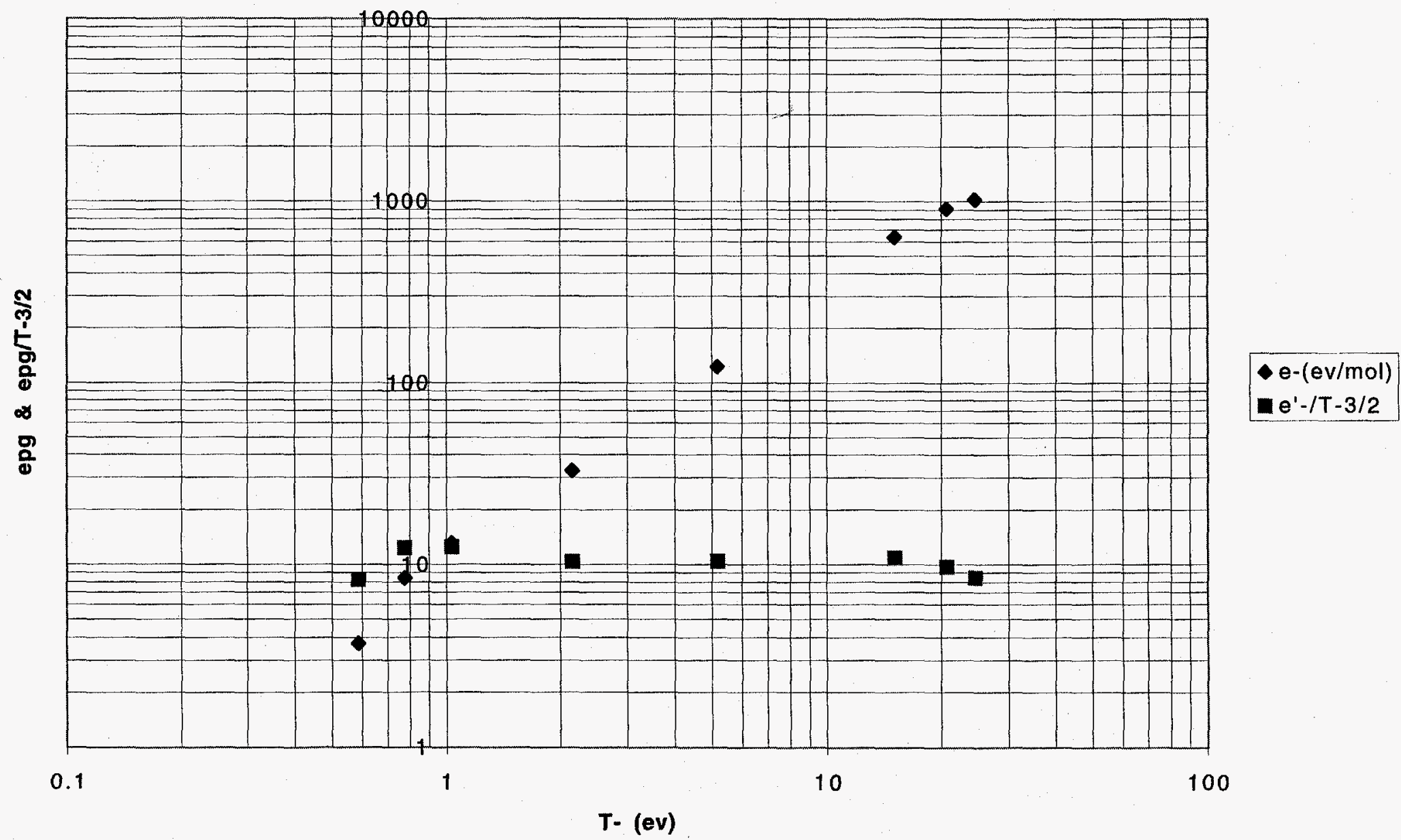


Fig. 5. Critical speed and temperature vs altitude

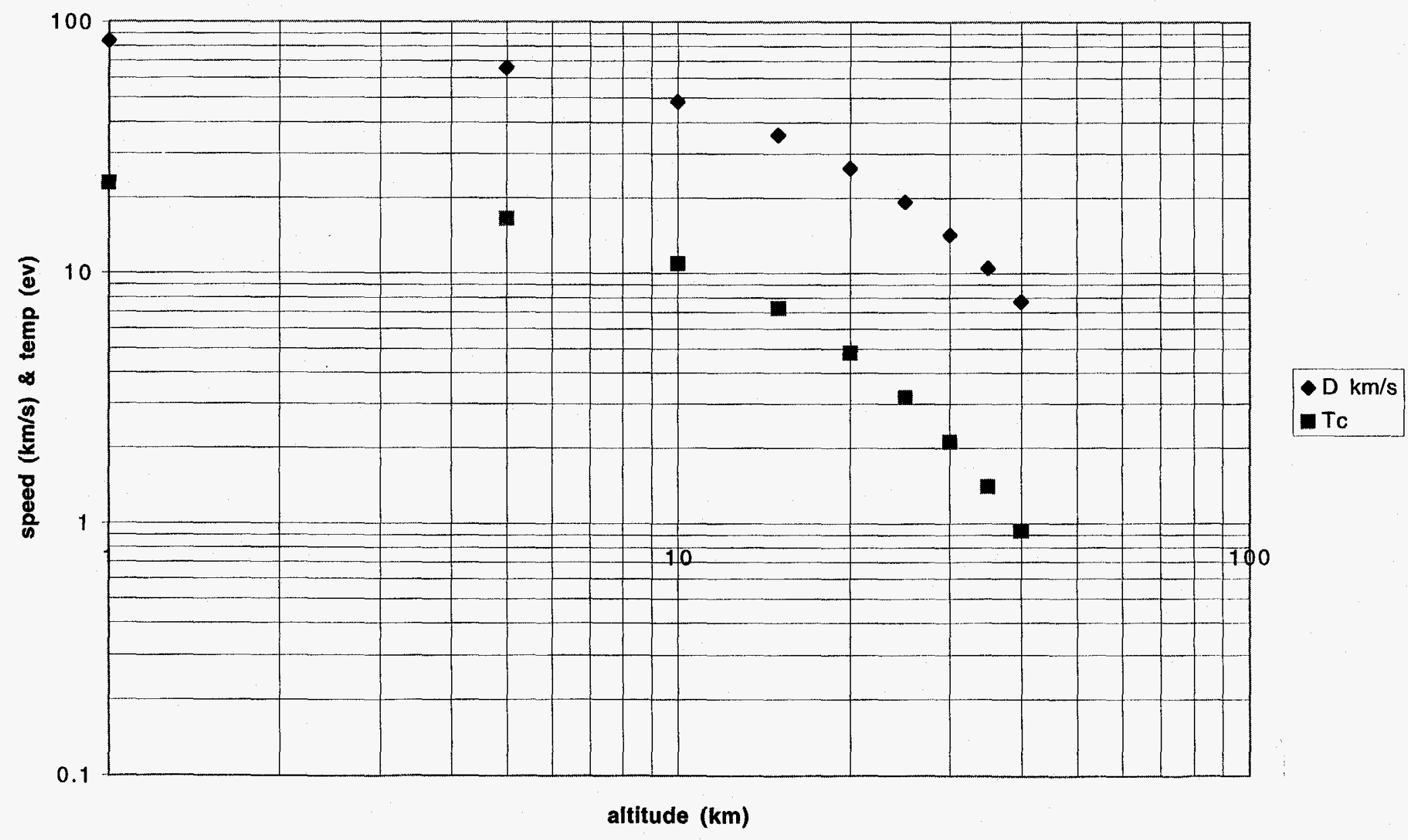


F6 norm flux $v \mathrm{~V}$

Fig. 6. Normalized fluxes vs shock speed

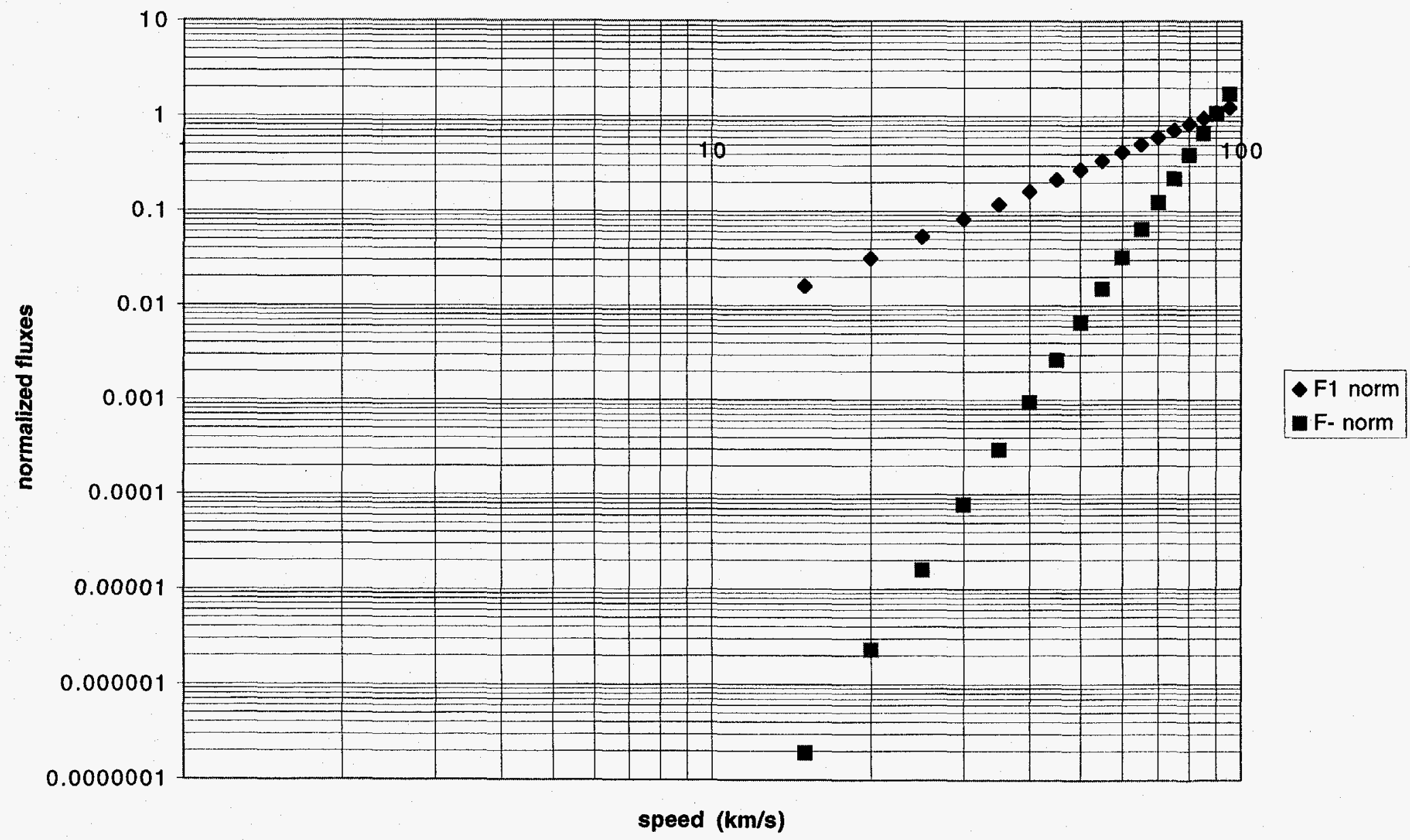

Page 1 
(

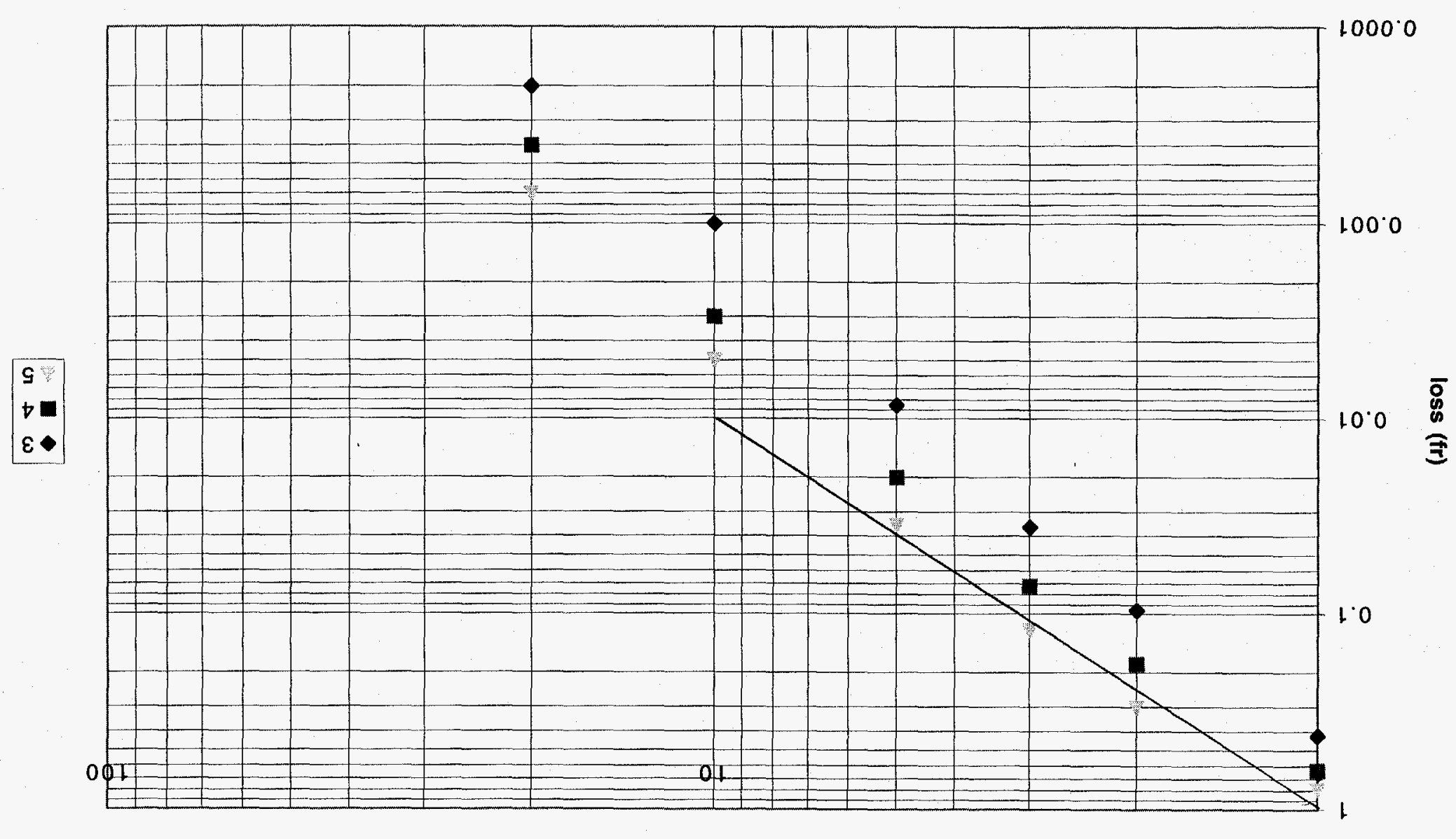

p|oysad4ł 
F8 shock rad v V

Fig. 8. Normalized fluxes vs velocity

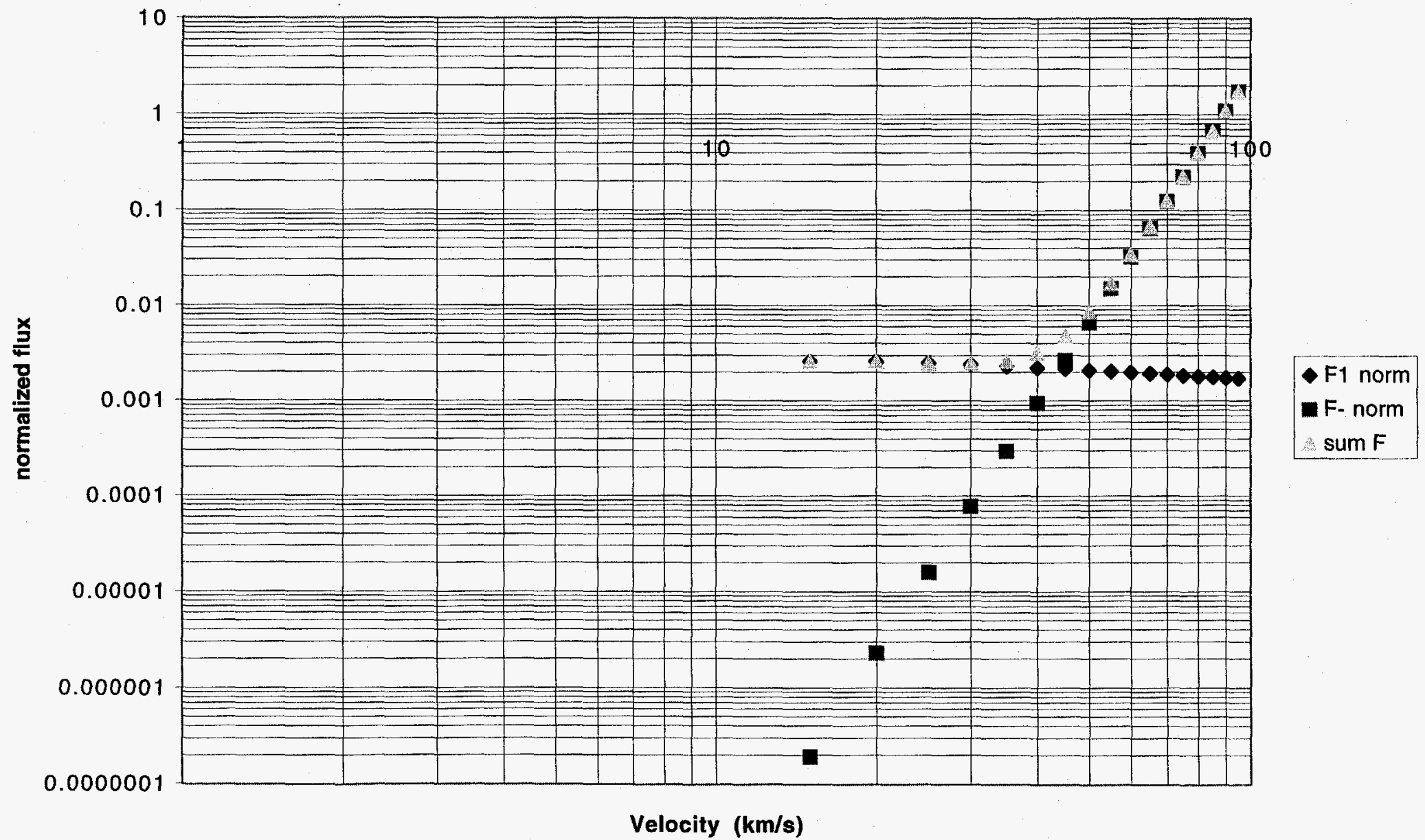


Fig. 9. Temp \& Tc vs altitude

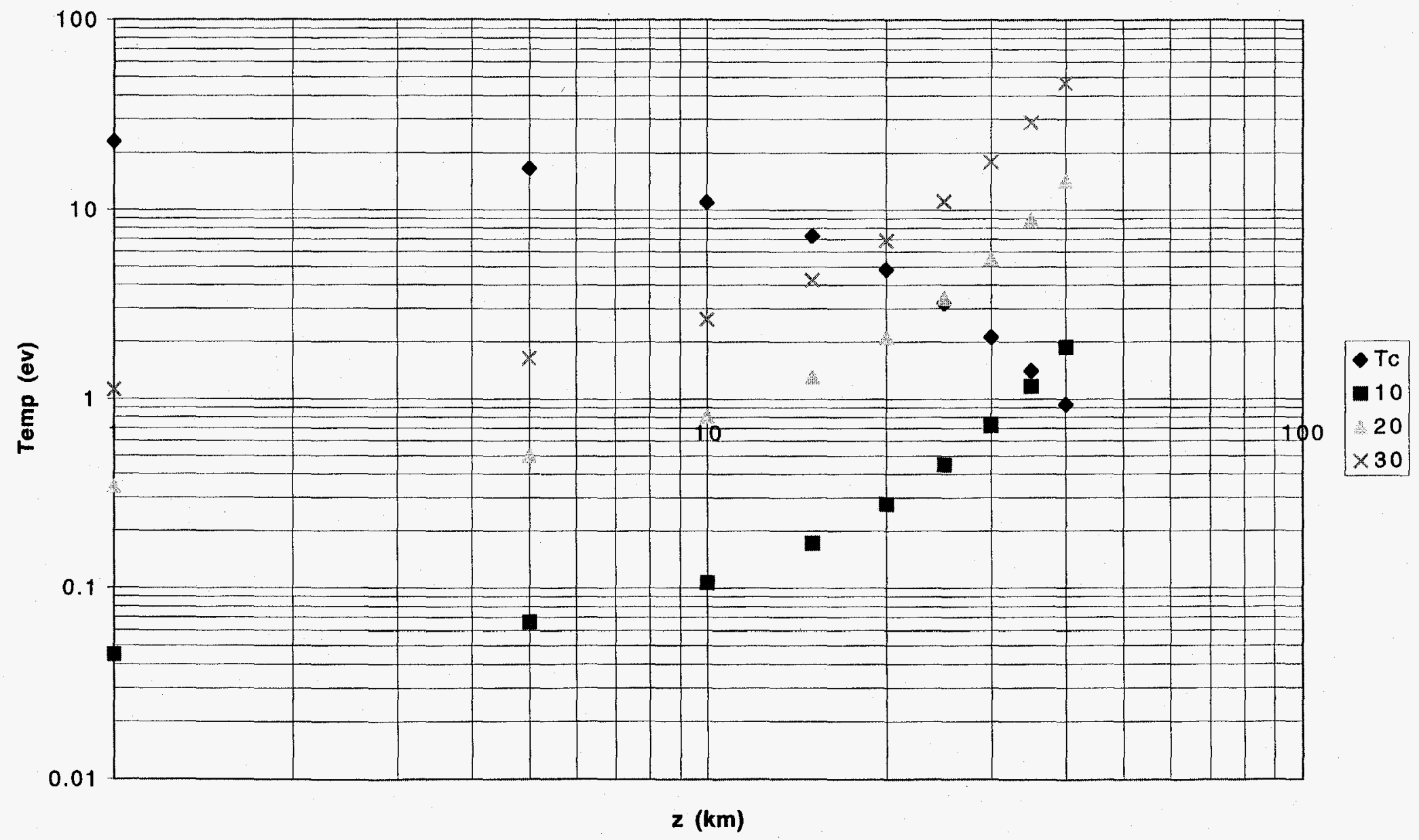


Fig. 10. Sub- \& Super-critical temperatures vs altitude

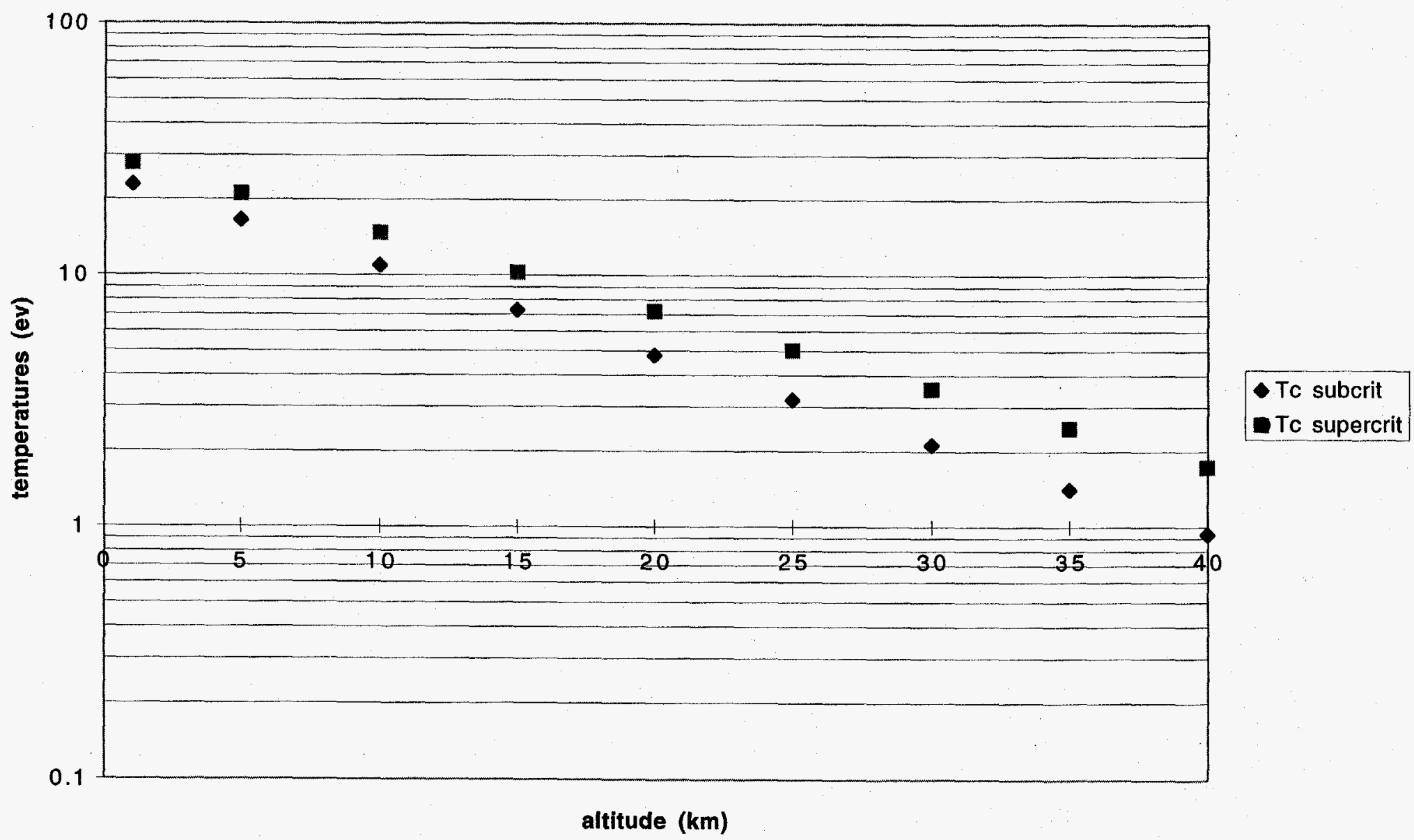


F11 limited $T \vee z$

Fig. 11. Limited temperatures vs altitude

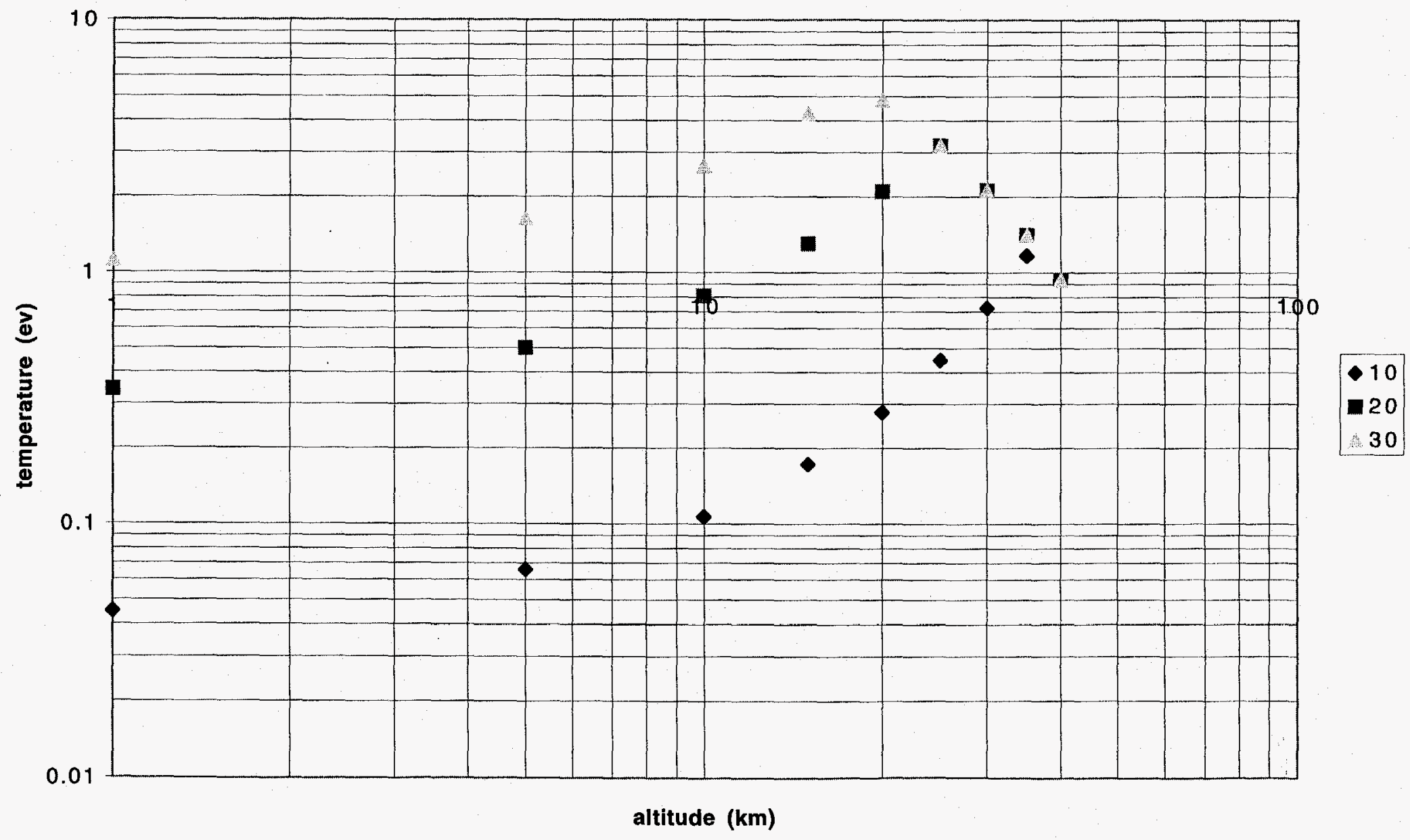

Page 1 


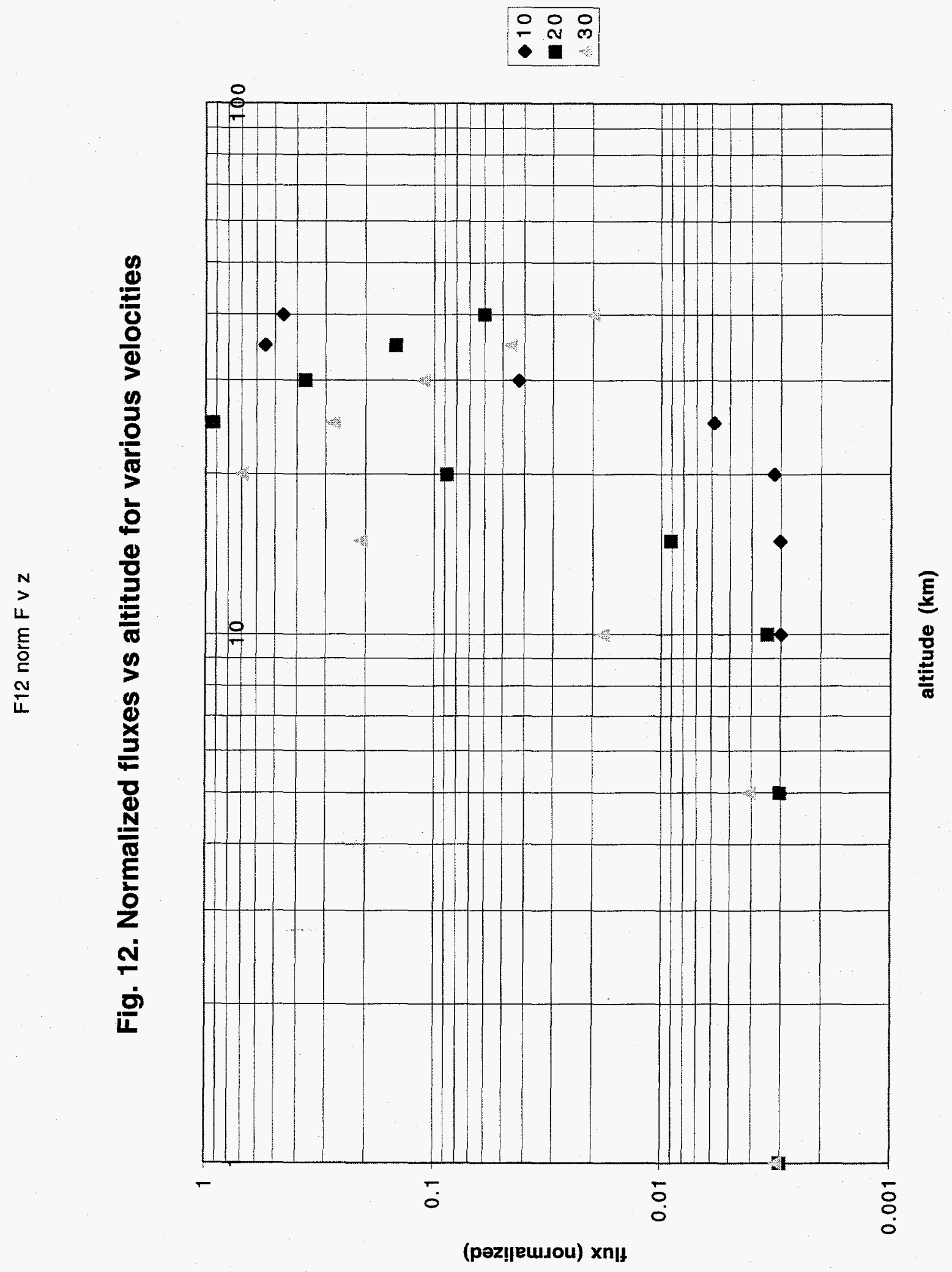




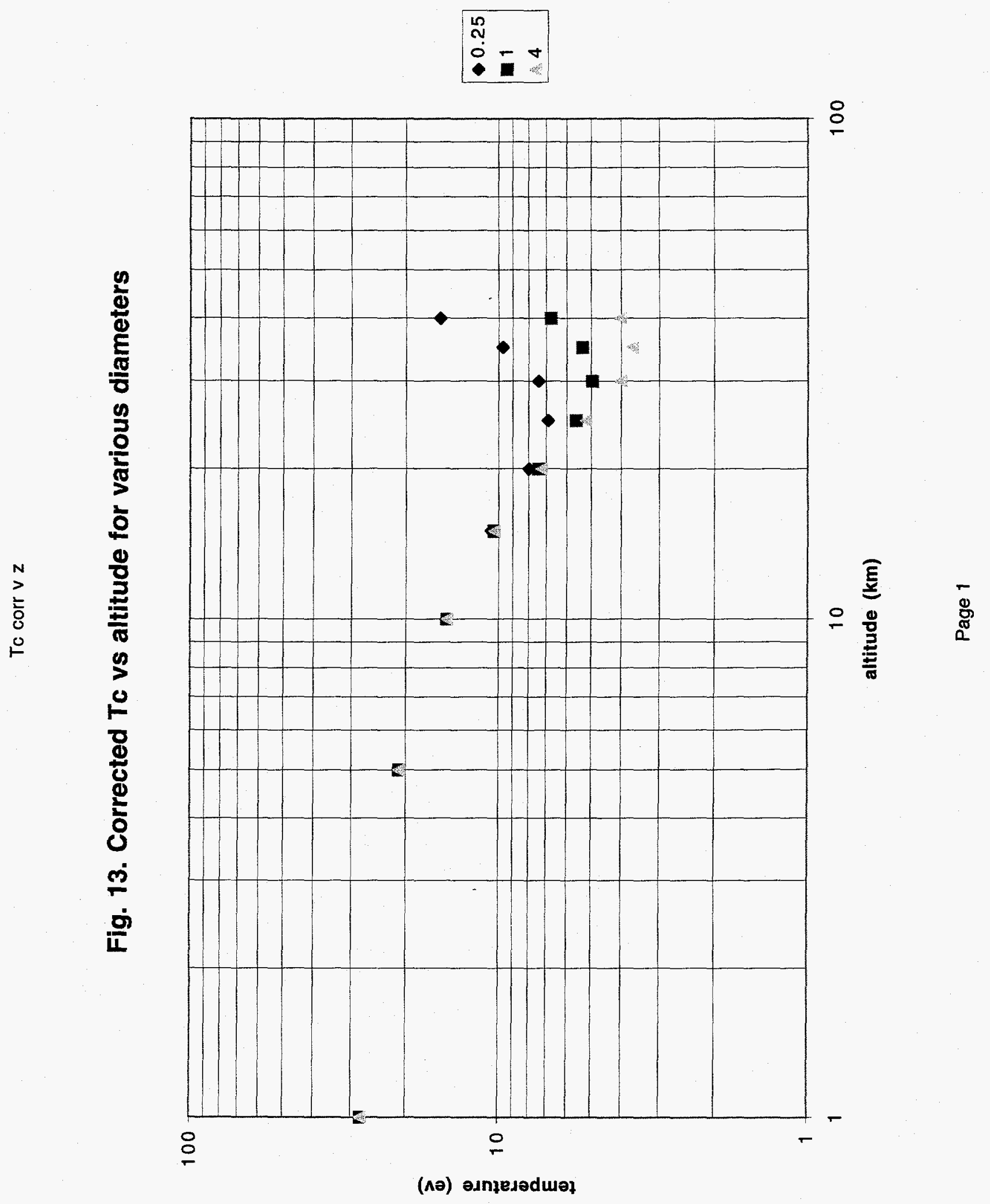


(யy) әрnұ!!|e

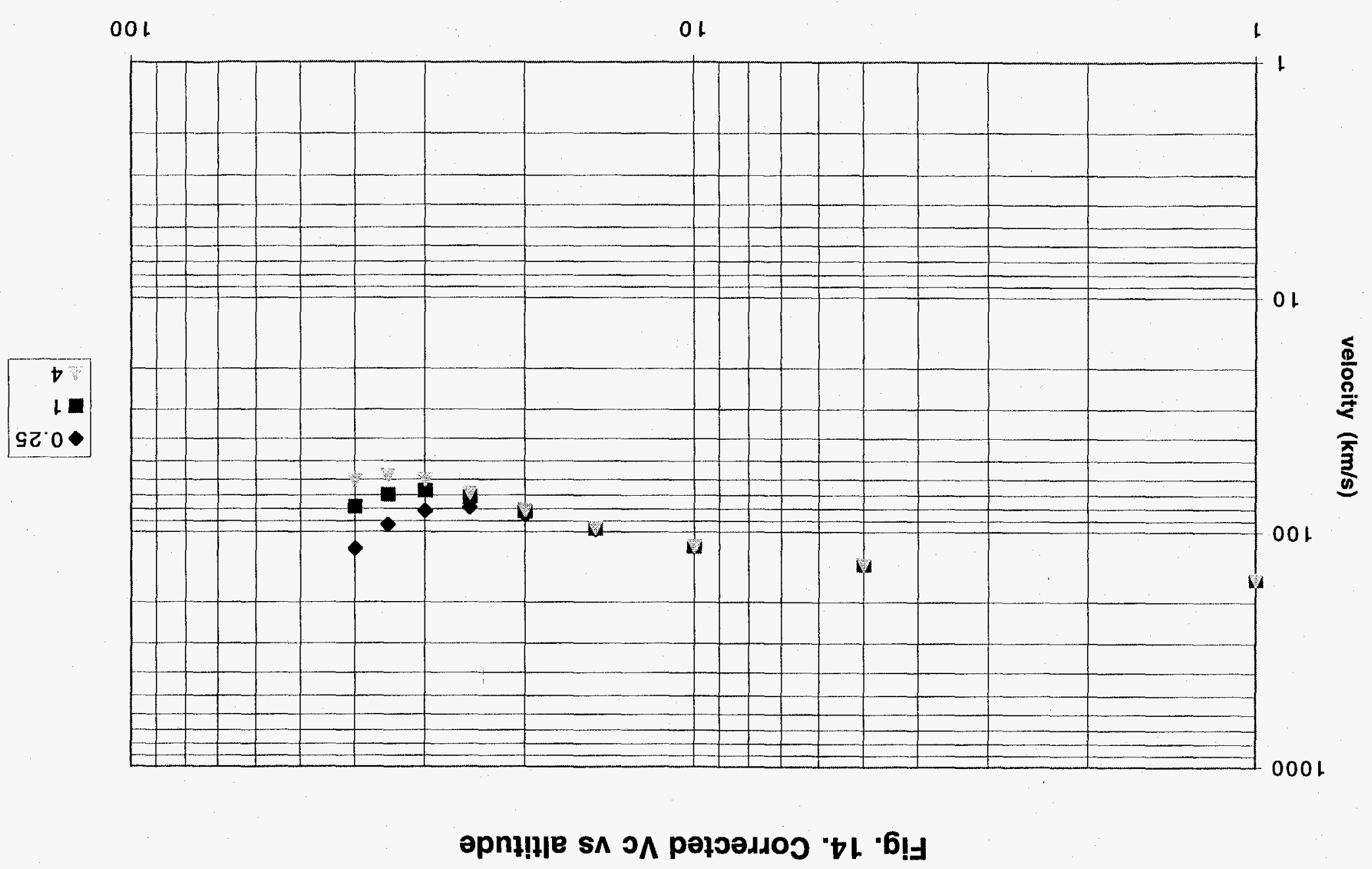

$z \wedge 11000 \wedge$ 


\section{(uy) әpnา!ฺ]e}

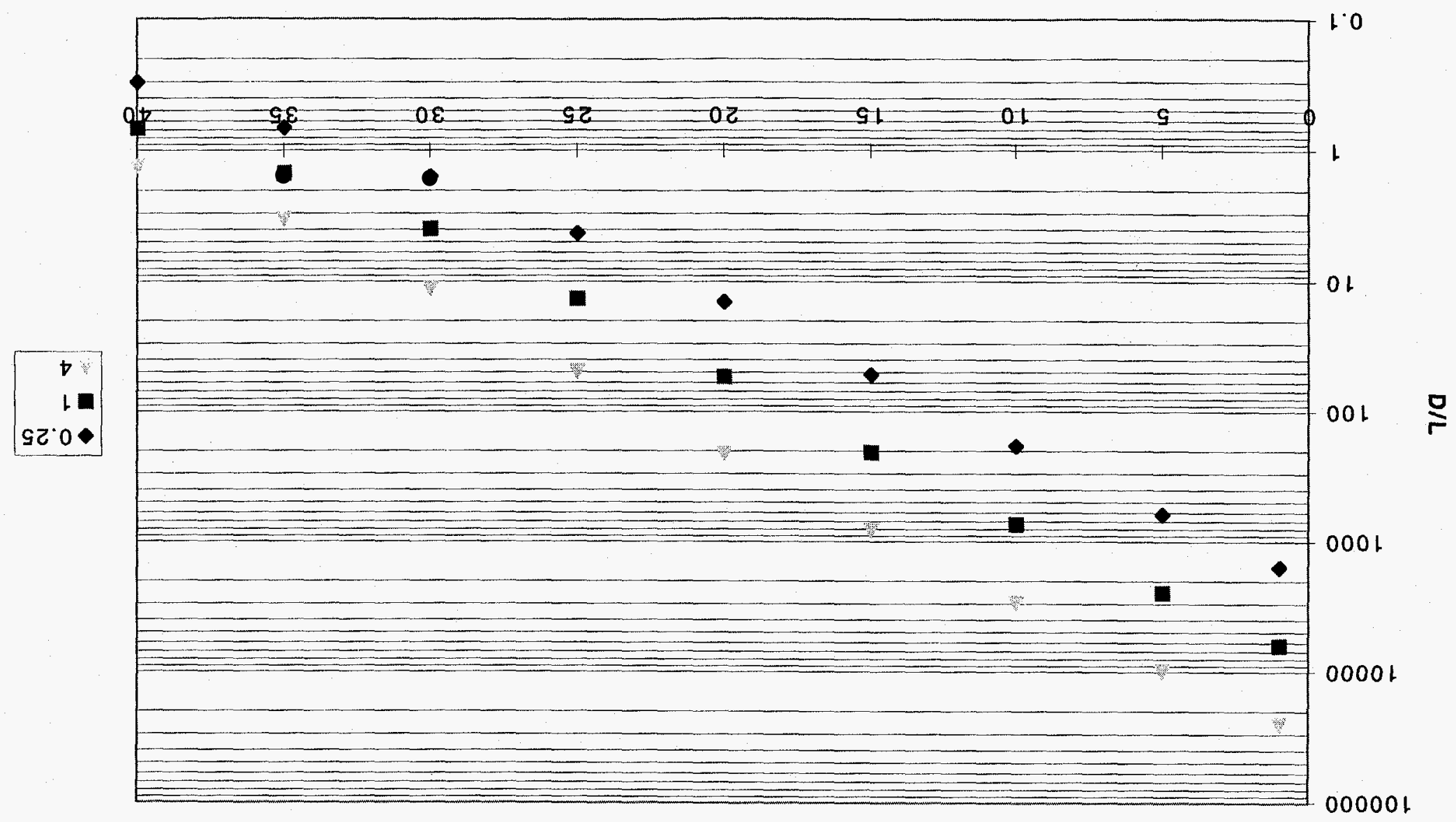

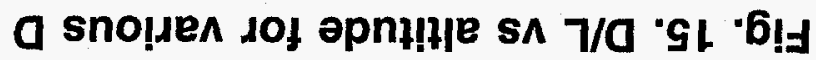

